\title{
Multicolor banding remains an important adjunct to array CGH and conventional karyotyping
}

\author{
Susan M Bint ${ }^{1 *}$, Angela F Davies ${ }^{1}$ and Caroline Mackie Ogilvie ${ }^{1,2,3}$
}

\begin{abstract}
Background: Array comparative genomic hybridization (CGH) for high resolution detection of chromosome imbalance, and karyotype analysis using G-banded chromosomes for detection of chromosome rearrangements, provide a powerful diagnostic armoury for clinical cytogenetics. However, abnormalities detected by karyotype analysis cannot always be characterised by scrutinising the G-banded pattern alone, and imbalance detected by array CGH cannot always be visualised in the context of metaphase chromosomes. In some cases further techniques are needed for detailed characterisation of chromosomal abnormalities. We investigated seven cases involving structural chromosome rearrangements detected by karyotype analysis, and one case where imbalance was primarily detected by array CGH. Multicolor banding (MCB) was used in all cases and proved invaluable in understanding the detailed structure of the abnormalities.

Findings: Karyotype analysis detected structural chromosome rearrangements in 7 cases and MCB was used to help refine the karyotype for each case. Array CGH detected imbalance in an eighth case, where previously, G-banded chromosome analysis had reported a normal karyotype. Karyotype analysis of a second tissue type revealed this abnormality in mosaic form; however, MCB was needed in order to characterise this rearrangement. MCB provided information for the delineation of small deletions, duplications, insertions and inversions and helped to assign breakpoints which were difficult to identify from G-banded preparations due to ambiguous banding patterns.

Conclusion: Despite the recent advance of array CGH in molecular cytogenetics we conclude that fluorescence in situ hybridization, including $M C B$, is still required for the elucidation of structural chromosome rearrangements, and remains an essential adjunct in modern diagnostic laboratories.
\end{abstract}

Keywords: Multicolor banding, Fluorescence in situ hybridization, Array CGH, Karyotype analysis, Complex chromosome rearrangements

\section{Background}

Multicolor banding (MCB) is a highly reliable and reproducible fluorescence in situ hybridization (FISH) technique that can be used for the detection of balanced and unbalanced chromosome rearrangements and to help characterize complex chromosome rearrangements and marker chromosomes [1,2] that cannot be characterized by array comparative genomic hybridization $(\mathrm{CGH})$ or by G-banded chromosome analysis and standard FISH techniques alone. Images are processed using specialised software (MetaSystems - Isis) which converts the fluorescence profiles along the length of the chromosome to

\footnotetext{
*Correspondence: susan.bint@gsts.com

'Cytogenetics department, GSTS-Pathology, Guy's and St. Thomas' Hospital NHS Foundation Trust, London SE1 9RT, UK

Full list of author information is available at the end of the article
}

pseudocolored bands [1,3]. These pseudocolored bands can then be compared to standard G-banded ideograms [4]. The Isis software enables selection of the number of pseudocolored bands on the chromosome to be analysed; it is therefore possible to achieve higher resolution with MCB than that using standard G-banding $[3,5]$.

Array CGH has replaced karyotype analysis as a "first line" genetic test for most referral indications in many laboratories [6-8]; however, it cannot determine the position of rearranged segments within the genome, or provide information on balanced rearrangements. Full characterisation of chromosome abnormalities is important in order to establish genotype-phenotype correlation, provide prognoses, and estimate reproductive risks for the proband and the family. 
We describe 8 cases where MCB has been used as an adjunct to karyotype analysis, routine FISH analysis and array $\mathrm{CGH}$ in order to characterise structural chromosome aberrations.

\section{Results}

Case 1

Referral reasons

Age 4 months - Hypotonia, obesity, developmental delay, mild/moderate MR and suspicion of Prader-Willi syndrome.

Age 16 years - no reading \& writing, simple speech, long face, right sided convergent squint, long tapering fingers, large hands and feet and overcrowded teeth.

\section{Preliminary karyotype}

$46, \mathrm{XX}, 12 \mathrm{p}+$

\section{Investigations}

Subtelomere Multiplex Ligation-dependent Probe Amplification (MLPA);

Fluorescence in situ hybridization (FISH) with a locusspecific probe for a locus within band 12p13.2;

MCB probe set for chromosome 12 (XCyte 12).

MLPA, using probes specific for loci within the short and long arm subtelomere regions of every chromosome, showed a deletion of two different probes for the subtelomere region of the chromosome 12 short arm $(0.170 \mathrm{Mb}$ and $0.287 \mathrm{Mb}$ ) from the telomere. No duplication or deletion of any other subtelomere region was apparent.

The MCB pattern on the abnormal chromosome 12 was informative for the band order and indicated an inverted duplication of the terminal region of the short arm (Figure 1).

\section{Final karyotype}

46,XX,inv dup del(12)(qter $\rightarrow$ p13.3::p13.3 $\rightarrow$ p12.3:) [9-11]

\section{Case 2}

\section{Referral reasons}

Age 8 months - axial hypotonia and delay in motor milestones.

Preliminary karyotype

46,XX,der(7)[39]/46,XX[13]

\section{Investigations}

Subtelomere FISH with probes specific for the short and long arm subtelomere regions of chromosome 7;

Locus-specific probe FISH;

MCB probe set for chromosome 7 (XCyte 7).

The MCB pattern on the abnormal chromosome 7 was informative for the band order, and showed a complex rearrangement involving an insertion event and a deletion of the regions $7 q 22 \rightarrow 7 q 32$ and $7 q 34 \rightarrow 7 q 36$ (Figure 2).

\section{Final karyotype}

46,XX,der(7)(pter $\rightarrow$ p22::p11.2 $\rightarrow$ q22::q32 $\rightarrow$ q34::p11.2 $\rightarrow$ p22:: $\mathrm{q} 36 \rightarrow \mathrm{qter})[39] / 46, \mathrm{XX}[13]$.

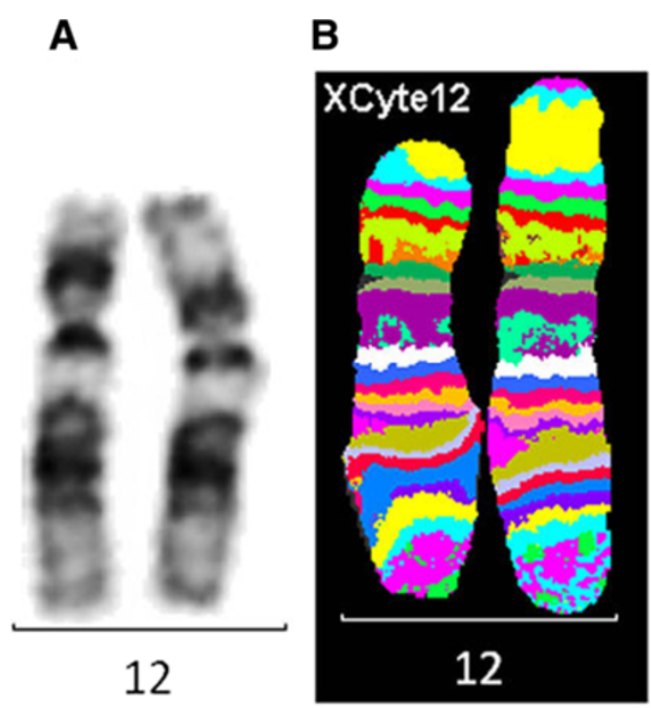

\section{C}
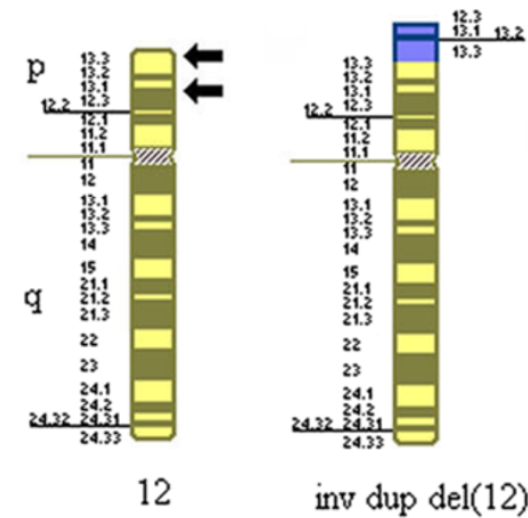

inv dup del(12)

Figure 1 Chromosome 12s for case 1. G-banded chromosome 12s (A), multicolor banded chromosome 12s (B) and partial G-banded ideogram (C) for case 1. Arrows denote breakpoints. 

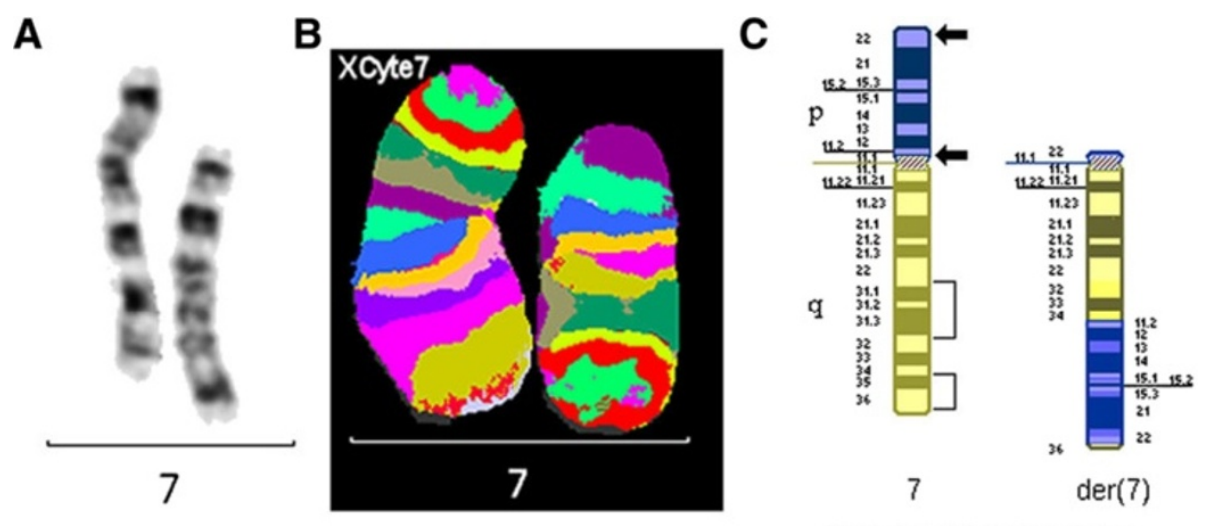

Key. ] = deleted regions

Figure 2 Chromosome 7s for case 2. G-banded chromosome 7s (A), multicolor banded chromosome 7s (B) and partial G-banded ideogram (C) for case 2. Please note that the arms of chromosome 7 are depicted in different colours, for clarity. Arrows denote breakpoints.

\section{Case 3}

Referral reasons

Age 1 month - cleft lip \& palate, hypotonia, depressed nasal bridge, flat occiput, hypoglycaemia and polyhydramnios in pregnancy.

\section{Preliminary karyotype}

$46, \mathrm{XX}, \operatorname{add}(7)(\mathrm{q} 22)$

\section{Investigations}

Whole chromosome paint specific for chromosome 7 (WCP7);

Partial chromosome paints specific for the short arm and long arm of chromosome 7 (PCP7p, PCP7q).

Microsatellite analysis

MCB probe set for chromosome 7 (XCyte 7).
The MCB pattern on the derivative chromosome 7 showed that the additional material present within the long arm of the abnormal chromosome was a duplication of the region $7 q 21.2 \rightarrow 7 q 22$ (Figure 3 ).

Final karyotype

46,XX,dup(7)(q21.2q22.1)

\section{Case 4}

\section{Referral reasons}

Age 11 years - mild learning difficulties, hypotonia, lax joints, hyperactive, large head, long face, ?Angelman syndrome.
A

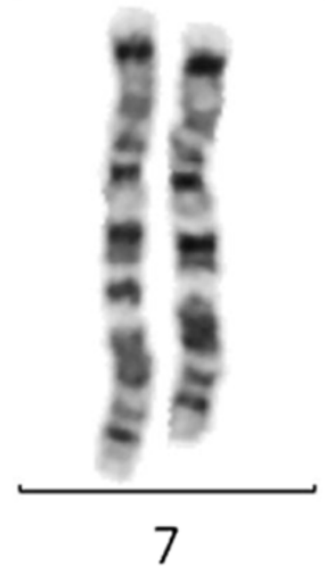

B

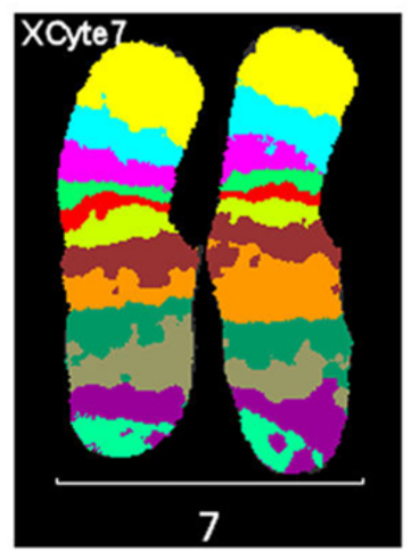

C

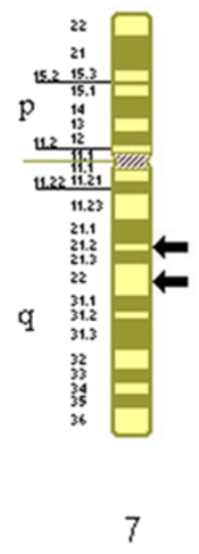

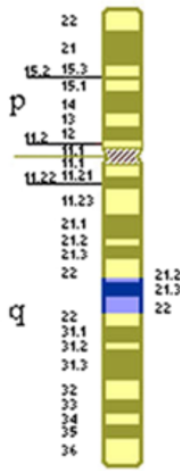

$\operatorname{dup}(7)$

Figure 3 Chromosome 7s for case 3. G-banded chromosome 7s (A), multicolor banded chromosome 7s (B) and partial G-banded ideogram (C) for case 3. Arrows denote breakpoints. 


\section{Investigations}

Multiwell whole chromosome paints (WCPs) for every chromosome;

MCB probe sets for chromosomes 13 and 18 (XCyte 13 \& XCyte 18 respectively).

These results together with those of the G-banded chromosome analysis showed that this patient had a paracentric inversion in the short arm of chromosome 2 ; the proximal two thirds of the chromosome
18 long arm $(18 \mathrm{q} 11.2 \rightarrow 18 \mathrm{q} 21.3)$ was directly inserted into the short arm of chromosome 7 and a central segment of the chromosome 7 short arm, comprising about two thirds of the short arm, was inverted and inserted into the chromosome 13 long arm at band 13q12.2 (Figure 4).

\section{Final karyotype}

46,XY,inv(2)(p13p21),der(7)del(7)(p13p21.2)

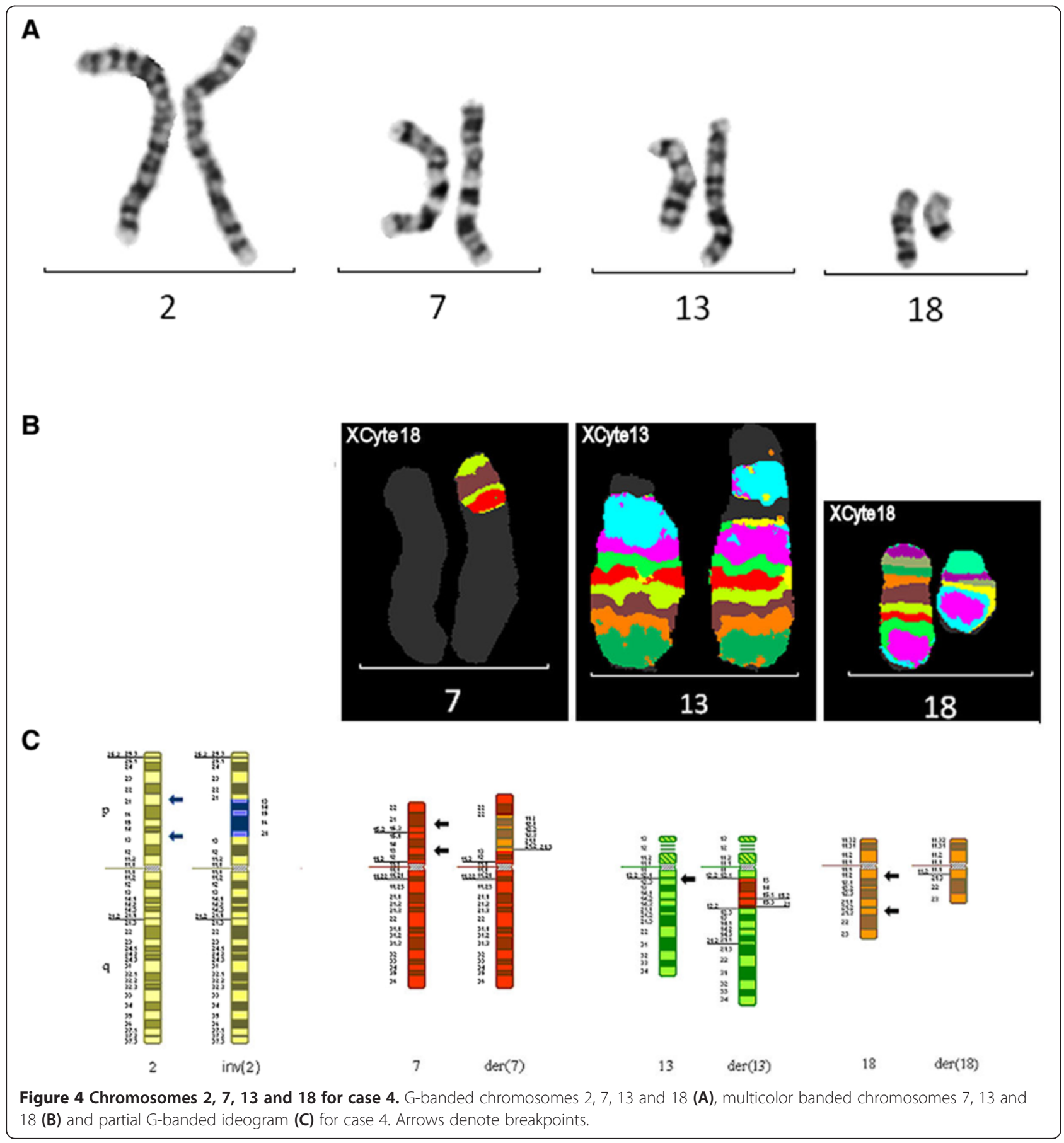


ins(7;18)(p13;q11.2q21.3),der(13) ins(13;7)(q12.2;p13p21.2), $\operatorname{der}(18) \operatorname{ins}(7 ; 18)(\mathrm{p} 13 ; \mathrm{q} 11.2 \mathrm{q} 21.3)$

\section{Case 5}

\section{Referral reasons}

Termination of pregnancy at 23 weeks gestation for ventriculomegaly, large bilateral facial clefts, elongated skull, receding lower jaw, bilateral cleft-lip and palate, preauricular skin tags and low-set ears.

\section{Preliminary karyotype}

46,XX,t(3;12;15)(3qter $\rightarrow 3 p 14.2:: 15 q 26.1 \rightarrow 15 q$ ter;

12 ?p?13.1 $\rightarrow 12$ ?q?14::12?p?13.1 $\rightarrow 12$ ?pter;

15 pter $\rightarrow 15$ q26.1::3p14.2 $\rightarrow$ 3p?ter::12q?14 $\rightarrow 12$ qter)

\section{Investigations}

Subtelomere FISH;

WCP3, WCP12, WCP15;

MCB probe set for chromosome 12 (XCyte 12).

The MCB pattern on the derivative chromosome 12 was informative for the band order, and showed monosomy of the region $12 \mathrm{p} 12.3 \rightarrow \mathrm{p} 13$ and the region $12 \mathrm{q} 13.2 \rightarrow 12 \mathrm{q} 14$ [12] (Figure 5).

\section{Final karyotype}

46,XX,t(3;12;15)(3qter $\rightarrow 3$ p14.2::15q26.1 $\rightarrow 15$ qter;12pter $\rightarrow$ 12p13.2::12q13.?2 $\rightarrow 12 \mathrm{p} 12.3:: 3 \mathrm{p} 26.3 \rightarrow$

3pter;15pter $\rightarrow 15$ q26.1::3p14.2 $\rightarrow$ 3p26.3::12q14 $\rightarrow 12$ qter)

\section{Case 6}

\section{Referral reasons}

Age 3 years - global developmental delay, speech \& language delay, poorly formed arches of feet, no obvious dysmorphic features.

\section{Preliminary karyotype}

46,XX,del(17)(q11.2q25.3),der(22)(22pter $\rightarrow 22 q 13.33::$

$17 q ? 25.3 \rightarrow 17 q$ ?11.2::17q25.3 $\rightarrow 17 q$ ter $)$

\section{Investigations}

Locus-specific FISH; MCB probe set for chromosome 17 (XCyte 17).

The FISH studies showed that probe specific for the long arm subtelomere region of chromosome 17 (D17S928, Abbott) hybridized to the terminal region of the derivative chromosome 22 and to the deleted chromosome 17. The most proximal subtelomere probe for chromosome 22 (D22S1056, Qbiogene (0.74Mb from the telomere)) hybridized to the derivative chromosome 22, while the most distal subtelomere probe (D22S1726, Cytocell (0.097Mb from the telomere)) did not hybridize to the derivative chromosome 22 .
The MCB pattern on the derivative chromosome 22 was informative for the band order, and indicated an inversion of the region $17 \mathrm{q} 11.2 \rightarrow \mathrm{q} 25$ (Figure 6).

\section{Final karyotype}

46,XX,del(17)(q11.2q25.3),der(22)(22pter $\rightarrow 22 q 13.33::$

$17 q 25.3 \rightarrow 17 q 11.2:: 17 q 25.3 \rightarrow 17 q t e r)$

\section{Case 7}

\section{Referral reasons}

Age 2 months - CHD - ASD/VSD, triangular face with frontal bossing and suspicion of DiGeorge syndrome.

\section{Initial karyotype}

46,XY,?dup(11)(p12p13)

\section{Investigations}

PCP11p, PCP11q;

Multiwell WCPs for all chromosomes;

Microsatellite analysis;

MCB probe set for chromosome 7 (XCyte7).

The MCB pattern showed that the chromosome 7 material present in the proximal region of the short arm of the abnormal chromosome 11 was derived from within the region $7 \mathrm{p} 11.2 \rightarrow 7 \mathrm{p} 13$. This $(7 \mathrm{p} 12.2 \rightarrow 7 \mathrm{p} 13)$ was inserted into the short arm of chromosome 11 at band $11 \mathrm{p} 13$. The orientation of the inserted segment of chromosome 7 into chromosome 11 could be determined from the G-banded chromosome pattern (Figure 7).

\section{Final karyotype}

46,XY,der(11)ins(11;7)(p13;p12.2p13)

\section{Case 8}

Referral reasons

Age 3 days - developmental delay, head lag, cleft palate, hypertelorism and left kidney dilation.

Age 6 days - ?mosaicism.

\section{Initial karyotype}

46,XY (peripheral blood sample)

\section{Further investigations due to abnormal phenotype}

Array CGH of blood sample:

arr 12p11.1p13.33(192,403-34,278,525)x3;

MLPA;

G-banded chromosome analysis of cultured fibroblasts detected a marker chromosome in $82.5 \%$ (33/40) of cells examined;

MCB probe set for chromosome 12 (Xcyte12).

The MCB pattern on the marker chromosome was informative for the band order.

These results confirmed that the marker chromosome was derived from the short arm of chromosome 12, and 
A

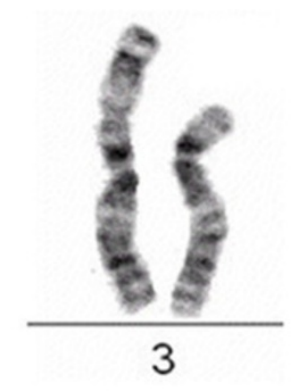

B

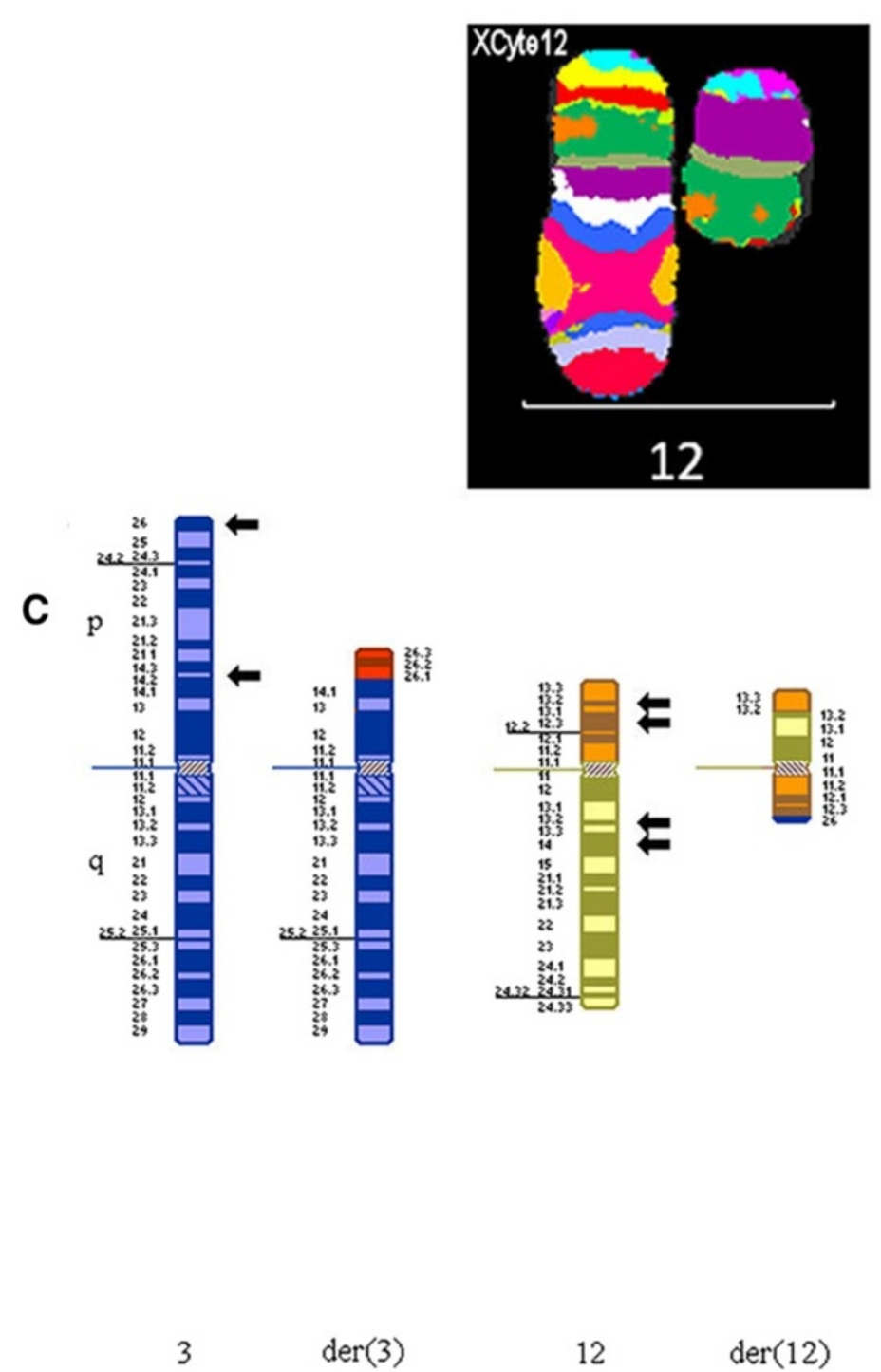

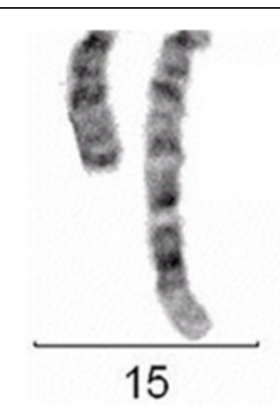

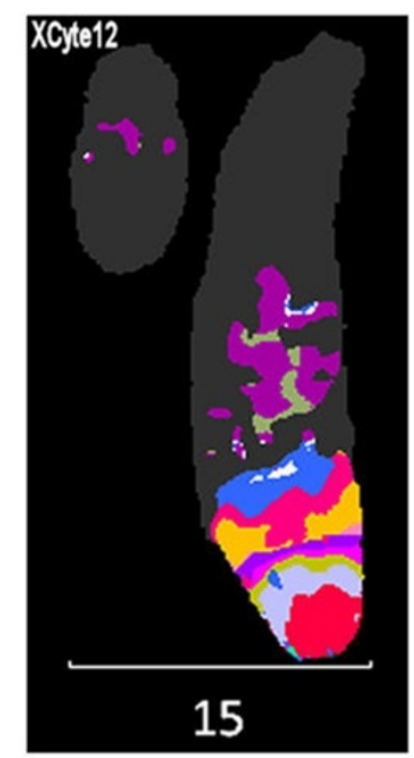

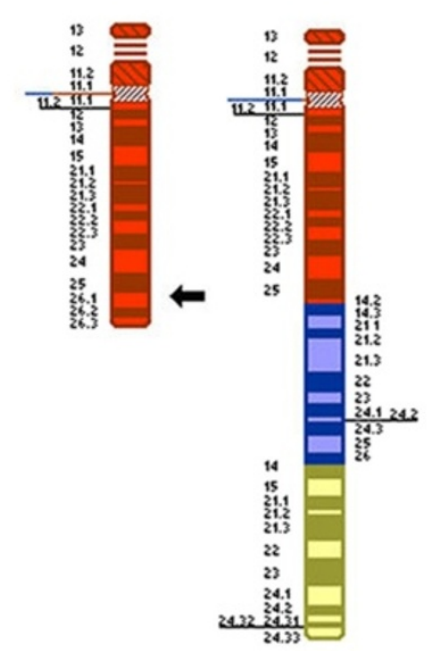

Figure 5 Chromosomes 3, 12 and 15 for case 5. G-banded chromosomes 3,12 and 15 (A), multicolor banded chromosomes 12 and 15 (B) and partial G-banded ideogram (C) for case 5. Arrows denote breakpoints. Please note that the arms of chromosome 12 are depicted in different colours, for clarity. 


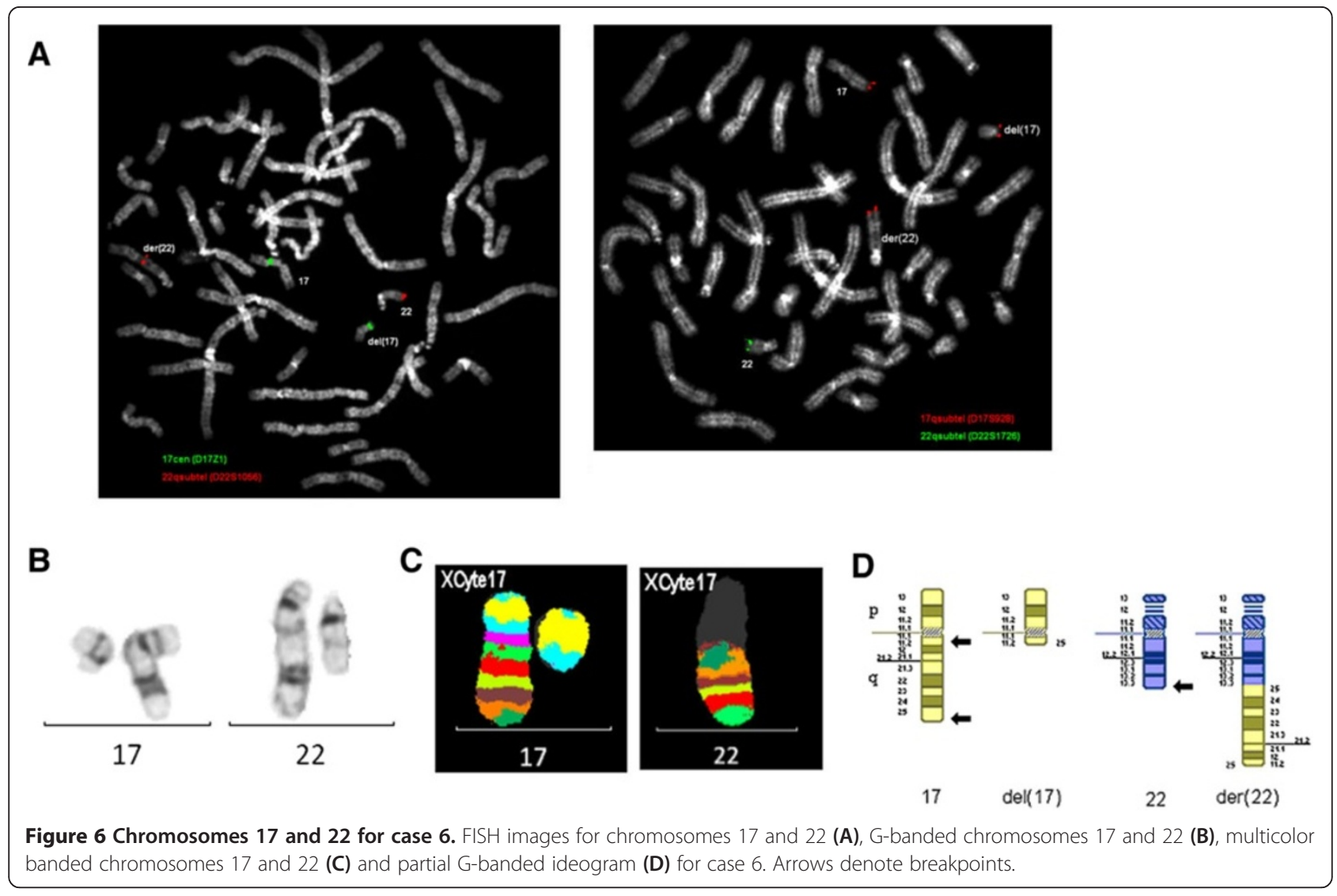

was not a typical $i(12 p)$, but was the result of a breakage/ inversion event (with breakpoints at the centromere and at band p13.1) with duplication of the derived product, giving a symmetrical pseudodicentric structure (Figure 8).

\section{Final karyotype}

47,XY,+der(12)(12pter $\rightarrow 12 p 13.1:: 12 p 10 \rightarrow 12 p 13.1: 12 p 13.1 \rightarrow$ 12 p10::12p13.1 $\rightarrow 12$ pter)[33]/46,XY[7]

\section{Discussion}

Multicolor banding is the most informative hybridization technique for characterization of chromosome rearrangements [12,13]. This is because, compared to WCP probes, $\mathrm{MCB}$ can provide specific information regarding the position of breakpoints and imbalance $[12,14,15]$. This information is invaluable for the clinical management of patients and for characterizing parental balanced chromosome rearrangements as it enables the

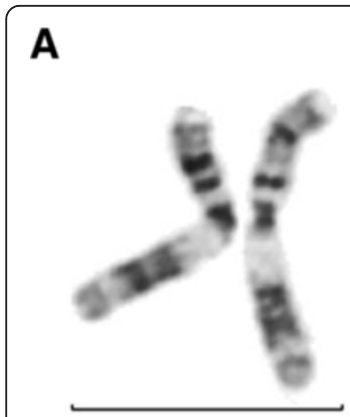

11
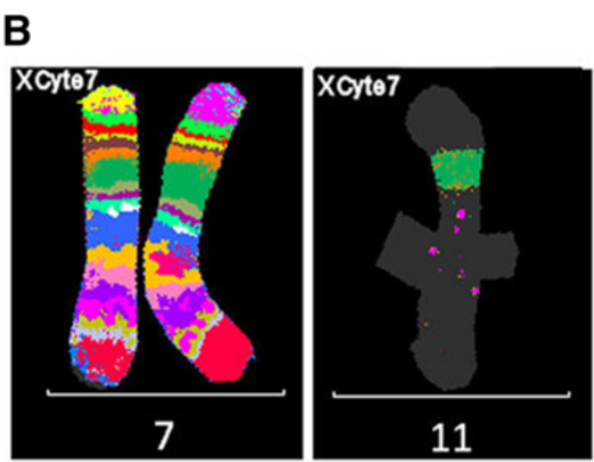

C

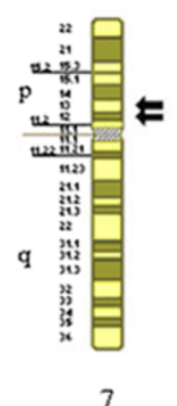

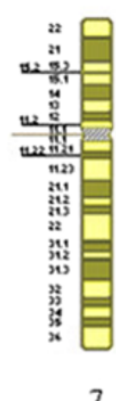

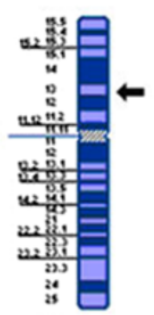

11

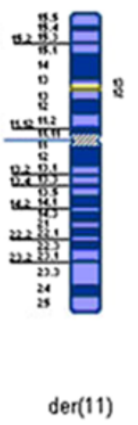

$\operatorname{der}(11)$

Figure 7 Chromosomes 7 and 11 for case 7. G-banded chromosome 11s (A), multicolor banded chromosomes 7 and 11 (B) and partial G-banded ideogram (C) for case 7. Arrows denote breakpoints. 


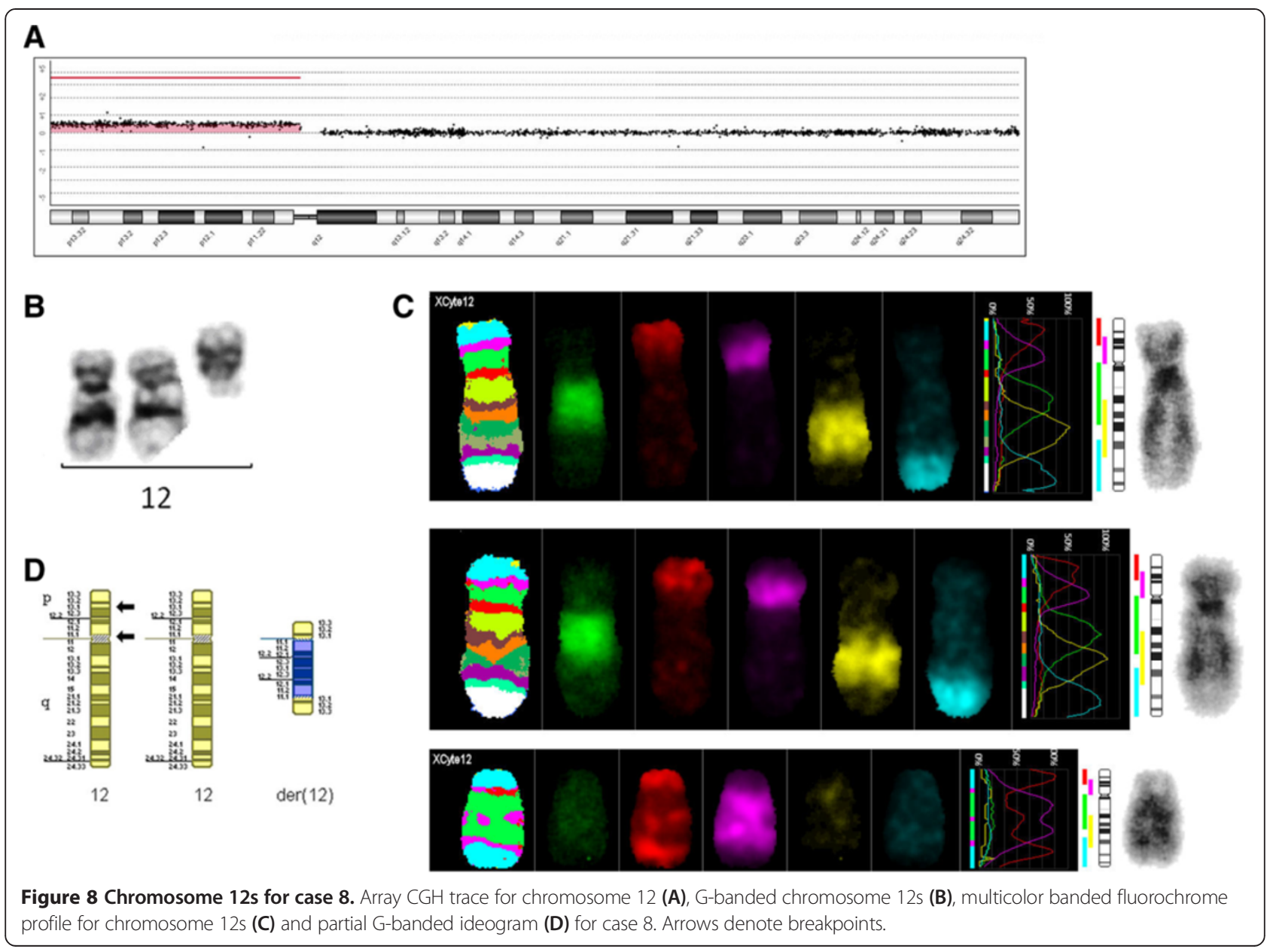

calculation of accurate reproductive risks for the parents for future pregnancies.

In this laboratory, $\mathrm{MCB}$ has been carried out on 8 cases that have undergone either karyotype analysis alone or karyotype analysis and array $\mathrm{CGH}$; in all cases, MCB provided information not available from the other tests carried out. We conclude that MCB is an important adjunct to array CGH and conventional karyotyping; for balanced rearrangements detected by karyotyping, MCB will provide more detailed breakpoint and orientation information, and for duplications detected by array $\mathrm{CGH}, \mathrm{MCB}$ will detect the genomic location and orientation of the additional material. Multicolor banding therefore still has an important place in modern diagnostic laboratories.

\section{Conclusions}

A modern cytogenetics laboratory should have access to a range of molecular cytogenetic tools, including various in situ hybridisation protocols. Of these, MCB is an important adjunct, which can provide information not available using other techniques.

\section{Methods}

\section{Karyotyping}

For all cases chromosomes were prepared from peripheral blood lymphocyte cultures following standard laboratory procedures. Case 8 also had chromosome preparations made from cultured fibroblasts. Analysis of G-banded chromosomes was carried out at a resolution of 400-550 bands per haploid genome using standard protocols.

\section{FISH}

In situ hybridization studies were carried out for each case using a range of locus specific, chromosome enumeration and subtelomere probes, partial chromosome paints (PCP) and whole chromosome paints (WCP).

\section{Multicolor banding}

Multicolor banding with a cocktail of microdissected region-specific overlapping partial chromosome paints (PCP) was carried out on metaphase chromosomes from peripheral blood samples for cases 1 to 7 and from cultured fibroblasts for case 8, according to the protocol recommended by MetaSystems $(\mathrm{GmbH}$, Altlussheim, 
Germany). The MCB probes used were specific to the chromosomes involved in the rearrangement and all were from Metasystems GmbH, Altlussheim, Germany. Cells were captured with a CCD camera and processed using the ISIS/mFISH imaging system (Metasystems, Altlussheim, Germany). Fluorescence intensity ratios produced by the region-specific PCPs along the longitudinal axis of the chromosome were used to assign pseudocolours to chromosomal regions by means of the ISIS software.

\section{MLPA}

MLPA was carried out on DNA extracted from peripheral blood for cases 1 and 8 using MLPA kits (P069, P036 or P036B, MRC-Holland, The Netherlands) containing probes for the subtelomere regions of every chromosome, as described previously [16].

\section{Microsatellite analysis}

Microsatellite analysis was carried out on DNA extracted from peripheral blood for cases 3 and 7 using polymorphic microsatellite markers specific for chromosome 7 as described previously [17]. Three markers were informative (D7S820 (7q21.11), D7S796 (7q22.1) and D7S2847 (7q31.31)) for case 3 and two markers specific for the short arm of chromosome 7 (D7S2552 within distal 7p11.2 and D7S628 (7p14.3)) were informative for case 7 .

\section{Array CGH}

Array CGH was carried out on DNA extracted from peripheral blood lymphocytes from case 8 as described previously [8], using an oligonucleotide array slide (AMADID 017457; Agilent Technologies, Santa Clara, CA) containing approximately 44,000 probes across the genome.

\section{Competing interests}

The authors declared that they have no competing interests.

\section{Authors' contributions}

SMB participated in the cytogenetic analysis and interpretation, drafted the manuscript and organized the revisions. AFD participated in the cytogenetic analysis and interpretation and critically revised the manuscript. CMO conceived of the study, participated in its design, helped to draft the manuscript and revised it critically. All authors read and approved the final manuscript.

\section{Acknowledgments}

We would like to thank Toby Kirby, Mariama Kabba, Remi Oke and Philippa May for carrying out the FISH and MCB technical work for some of the cases.

\section{Author details}

'Cytogenetics department, GSTS-Pathology, Guy's and St. Thomas' Hospital NHS Foundation Trust, London SE1 9RT, UK. 'enetics Centre, Guy's and St Thomas' NHS Foundation Trust, London SE1 9RT, UK. 'Division of Genetics and Molecular Medicine, King's College London, School of Medicine at Guy's, King's College and St Thomas' Hospitals, London SE1 9RT, UK.

\section{References}

1. Chudoba I, Plesch A, Lorch T, Lemke J, Claussen U, Senger G: High resolution multicolor-banding: a new technique for refined FISH analysis of human chromosomes. Cytogenet Cell Genet 1999, 84:156-160.

2. Liehr T, Heller A, Starke H, Rubtsov N, Trifonov V, Mrasek K, Weise A, Kuechler A, Claussen U: Microdissection based high resolution multicolor banding for all 24 human chromosomes. Int J Mol Med 2002, 9:335-339.

3. Liehr T, Starke H, Weise A, Lehrer H, Claussen U: Multicolor FISH probe sets and their applications. Histol Histopathol 2004, 19:229-237.

4. Shaffer LG, McGowan-Jordan J, Schmid M (Eds): ISCN (2013): an international system for human cytogenetic nomenclature. Basel: Karger; 2013.

5. Liehr T, Weise A, Heller A, Starke H, Mrasek K, Kuechler A, Weier HU, Claussen U: Multicolor chromosome banding (MCB) with YAC/BAC-based probes and region-specific microdissection DNA libraries. Cytogenet Genome Res 2002, 97:43-50.

6. Lu X, Shaw CA, Patel A, Li J, Cooper ML, Wells WR, Sullivan CM, Sahoo T, Yatsenko SA, Bacino CA, et al: Clinical implementation of chromosomal microarray analysis: summary of 2513 postnatal cases. PLoS One 2007 2:e327.

7. Park SJ, Jung EH, Ryu RS, Kang HW, Ko JM, Kim HJ, Cheon CK, Hwang SH, Kang HYCP: Clinical implementation of whole-genome array CGH as a first-tier test in 5080 pre and postnatal cases. Mol Cytogenet 2011, 4:12.

8. Ahn JW, Bint S, Bergbaum A, Mann K, Hall RP, Mackie Ogilvie C: Array CGH as a first line diagnostic test in place of karyotyping for postnatal referrals - results from four years' clinical application for over 8,700 patients. Mol Cytogenet 2013, 6:16.

9. Zelante L, Calvano S, Dallapiccola B, Mingarelli R, Antonacci R, Chiovato L, Rocchi M: Patient with de novo $12 \mathrm{p}+$ syndrome identified as dir dup (12) (p13) using subchromosomal painting libraries from somatic cell hybrids. Clin Genet 1994, 46:368-71.

10. Rauch A, Trautmann U, Pfeiffer RA: Clinical and molecular cytogenetic observations in three cases of "trisomy 12 p syndrome". Am J Med Genet 1996, 63:243-9.

11. Segel R, Peter I, Demmer LA, Cowan JM, Hoffman JD, Bianchi DW: The natural history of trisomy 12p. Am J Med Genet A 2006, 140:695-703.

12. Seller MJ, Bint S, Kavalier F, Brown RN, Ogilvie CM: Multicolor banding detects a complex three chromosome, seven breakpoint unbalanced rearrangement in an ICSI-derived fetus with multiple abnormalities. Am J Med Genet A 2006, 140:1102-1107.

13. Johannes C, Chudoba I, Obe G: Analysis of X-ray-induced aberrations in human chromosome 5 using high-resolution multicolour banding FISH (mBAND). Chromosome Res 1999, 7:625-633.

14. Chudoba I, Hickmann G, Friedrich T, Jauch A, Kozlowski P, Senger G: mBAND: a high resolution multicolor banding technique for the detection of complex intrachromosomal aberrations. Cytogenet Genome Res 2004, 104:390-393.

15. Hu J, Sathanoori M, Kochmar SJ, Surti U: Application of multicolor banding for identification of complex chromosome 18 rearrangements. J Mol Diagn 2006, 8:521-525. quiz 528.

16. Ahn JW, Mann K, Docherty Z, Mackie Ogilvie C: Submicroscopic chromosome imbalance in patients with developmental delay and/or dysmorphism referred specifically for Fragile $\mathrm{X}$ testing and karyotype analysis. Mol Cytogenet 2008, 1:2

17. Mann K, Fox SP, Abbs SJ, Yau SC, Scriven PN, Docherty Z, Ogilvie CM: Development and implementation of a new rapid aneuploidy diagnostic service within the UK National Health Service and implications for the future of prenatal diagnosis. Lancet 2001, 358:1057-1061.

doi:10.1186/1755-8166-6-55

Cite this article as: Bint et al:: Multicolor banding remains an important adjunct to array CGH and conventional karyotyping. Molecular Cytogenetics 2013 6:55. 